\title{
LA REVOLUCIÓN KANTIANA DE KUHN
}

Carlos SOLÍS SANTOS

Hace más de doscientos años que Kant expuso la insuficiencia del empirismo por desconocer que los objetos del conocimiento deben constituirse sometiéndose al aparato cognitivo del sujeto. Su particular Revolución Copernicana consistió en reconocer "que la misma experiencia constituye un tipo de conocimiento que requiere entendimiento y éste posee unas reglas que yo debo suponer en mí ya antes de que los objetos me sean dados, es decir, reglas a priori. Estas reglas se expresan en conceptos a priori a los que, por tanto, se conforman necesariamente todos los objetos de la experiencia y con los que deben concordar" (B xvii-xviii).

Ha pasado mucho tiempo y, aunque la revolución copernicana de Kant parece un logro estable de la filosofía occidental, la revolución kantiana de Kuhn ha transformado notablemente la concepción del componente a priori del conocimiento científico. En efecto, para Kuhn, quien definió su filosofía madura como kantismo post-darwinista ${ }^{1}$, los elementos a priori de la ciencia no provienen de un sujeto genérico, trascendental, más allá de los sujetos empíricos, psicológicos, sociológicos e históricos. Las taxonomias léxicas, en las que su libro largamente esperado y nunca publicado hace descansar el elemento a priori del conocimiento, no son necesarias ni constantes; "pueden cambiar y de hecho cambian tanto con el paso del tiempo como con el tránsito de una comunidad a otra" (ibidem).

1 "The Road Since Structure", en A. Fine, M. Forbes y L. Wessel, Proceedings of the 1990 Biennial Meeting of the Philosophy of Science Association, East Lansing: PSA, 1991, Vol. 2: 3-13; pág. 12.

Éndoxa: Series Filosoficas, $n^{\ominus} 9,1997$, UNED, Madrid:

Carlos Solís Santos: La reoolución kantiana de Kuhn. pp. 5-30. 
No ha pasado el tiempo, sin embargo, para la realidad independiente del sujeto. Seguimos sin poder decir nada de ella. Las dificultades neokantianas con la inefable cosa en sí siguen con nosotros, especialmente por la proclividad de Kuhn a conferirle una función causal en la permanencia y estabilidad del mundo fenoménico; por su tendencia a identificarla, por más que esté fuera del Espacio y el Tiempo no menos que de la categoría de causalidad, con "el todo del que se han fabricado tanto las criaturas como sus nichos, tanto el mundo 'interno' como el 'externo'" (Ibidem).

A pesar de esta incorporación de la realidad en sí al intercambio causal con la ciencia y sus a priori locales, el pensamiento de Kuhn está a un paso del deslizamiento hacia el idealismo y constructivismo social del conocimiento científico. Muchas declaraciones ambiguas suyas se han entendido así. Sin ambigüedades y con fruición, los sociólogos del programa fuerte han dado ese paso desde que en los años setenta el kuhnianismo saltó a la fama. Para ellos, tras la disolución y multiplicación cultural de las condiciones a priori de la experiencia científica, la cosa en sí no sería el límite único del conocimiento, sino que se desmembraría en múltiples límites, tan afectados por la cultura local como los marcos conceptuales del pensamiento comunitario. ${ }^{2}$

La indefinición de algunas tesis de Kuhn y la problemática coherencia de sus implicaciones, la distancia entre sus declaraciones de principio en favor de la objetividad, por un lado, y las consecuencias subjetivistas de sus análisis, por otro, no han hecho sino reflejar la tensión entre racionalismo y escepticismo que compartimos con la situación a que se enfrentaba Kant. La gran diferencia entre aquélla coyuntura y la nuestra, sin embargo, radica en que entre tanto se han constituido las ciencias sociales como la psicología y la sociología, no menos que la biología

${ }^{2}$ Así se interpreta el recurso de los científicos a estrategias realistas. Cf. B. Barnes, D. Bloor y J. Henry, Scientific Knowledge. A Sociological Analysis, Londres: Athlone, 1996, capítulo 4. 
evolutiva que. ha "naturalizado" en mayor o menor medida la epistemología. En cualquier caso, y contra un trasfondo anglosajón inconfundible, la filosofía de Kuhn posee una rancia raigambre europea continental que explica la novedad y originalidad de su pensamiento en el contexto cultural estadounidense al incorporar actitudes filosóficas neokantianas, concepciones discontinuistas tomadas de la tradición de la historia de la filosofía y una interpretación naturalista de las categorías kantianas.

Kuhn ha ofrecido bosquejos de su desarrollo intelectual en los prefacios a La estructura (1962) y a La tensión esencial (1977). Trabajó como físico en Harvard entre 1943 (B.S.) y 1946 (A.M.) y presentó su tesis en 1949. Pero en 1947 participó en uno de los cursos de ciencia para no científicos organizados por J.B. Conant y descubrió las revoluciones científicas bajo la forma concreta de la discontinuidad de la física de Galileo y Newton respecto a su antecesora, la física aristotélica. Sus concepciones ingenuas sobre el desarrollo acumulativo de la ciencia, sacadas de la cultura de los físicos y de su afición a la filosofía de la ciencia entonces vigente, sufrieron un descalabro. Entonces se entregó a la historia de la ciencia que lo había sacudido del sueño positivista.

La oportunidad para cambiar de profesión vino con su elección como junior fellow de la Society of Fellows de Harvard entre 1948 y 1951. Era ésta una institución académica que, lejos de ofrecer un título, desanimaba a estudiar para conseguir uno. Pretendia más bien que las personas prometedoras tuviesen la oportunidad de seguir su camino sin someterse a las estrecheces de la especialización. En este medio, Kuhn pudo leer sobre historia de la ciencia, que entonces era un producto con un fuerte sabor francés y en muchos casos con un tinte marcadamente filosófico, por no decir metafísico. Los aires parisienses vinieron servidos por personas como A. Koyré, L. Brunschvicg, E. Meyerson y H. Metzger, sobrina de Lévy-Bruhl. Asimismo leyó las historias de A.O. Lovejoy y de 
A. Maier. También pudo explorar un tanto al azar cualesquiera temas relacionados con sus preocupaciones. De este modo descubrió la relevancia de la psicología evolutiva de Piaget y la de la psicología de la Forma de W. Köhler y K. Koffka, no menos que el enfoque antipositivista y colectivista de L. Fleck.

Entre 1951 y 1958 inició su carrera académica como historiador de la ciencia en Harvard y en Berkeley y publicó su primer libro de historia sobre La revolución copernicana (1957). La siguiente ruptura vino en 1958-59, cuando pasó una temporada en el Center for Advanced Study in the Behavioral Sciences de Stanford. La primera "ruptura" se había producido en el verano de 1947 con el descubrimiento de las revoluciones científicas. Después de una década, Kuhn estaba perfectamente preparado para redactar el capítulo de las revoluciones para La estructura; pero entonces se le planteó el problema de cómo caracterizar el consenso que se produce entre revoluciones, en ausencia del menor rastro de enunciados compartidos ni de la aceptación explícita de definiciones de los términos cruciales. La segunda "ruptura" (según cuenta Merton, los miembros del Center se saludaban por las mañanas con un "¿qué, alguna ruptura hoy?") consistió en descubrir que lo que en realidad cumplía la función atribuida a las definiciones y reglas de correspondencia era el aprendizaje de ejemplos selectos de cómo resolver problemas. Como es sabido, estos paradigmas (en sentido estricto y restringido) fueron cruciales para "psicologizar" el procesamiento científico de la información y para "sociologizar" el uso colectivo de marcos a priori en la investigación científica. Tras esta segunda ruptura, el resto de la redacción de La estructura procedió sin mayores dificultades. Desde este momento, Kuhn se dedicó cada vez menos a la historia de la ciencia para centrarse en la filosofía, aunque todavía habría de colaborar a mediados de los sesenta en las Sources for the History of Quantum Physics y publicar La teoria del cuerpo negro (1978). En lo que sigue esbozaré las influencias continentales europeas en la génesis de la filosofía de Kuhn. 


\section{Kuhn entre los franceses}

Kuhn ha insistido ${ }^{3}$ en su deuda con los historiadores de la ciencia procedentes de la historia de las ideas filosóficas, como Lovejoy y sobre todo A. Koyré. La procedencia de la filosofía enseñó a estas personas a tratar globalmente los sistemas pretéritos como visiones holistas del mundo, coherentes en sí mismas e incompatibles (inconmensurables) con la nuestra, en contra de las tendencias positivistas de los Sarton y Mieli a ver el desarrollo de la ciencia como el descubrimiento pieza a pieza de los hechos y las leyes actualmente aceptados, sin discontinuidades ni rupturas salvo con los "errores". Reconoce también su deuda con los filósofos neokantianos que acudían a la historia de la ciencia en busca de las condiciones impuestas por la mente humana al conocimiento científico, como L. Brunschvicg y E. Meyerson, aunque más por "lo que vieron en los materiales históricos que por su filosofía" (ibid., pág. xv) que resultaba demasiado ajena en el contexto norteamericano.

La filosofía de la ciencia francesa del cambio de siglo, de raigambre post-kantiana, compartía con el Kuhn de la primera "ruptura", que se enfrentaba a finales de los cuarenta a las sorpresas de la historia de la ciencia, el disgusto con una u otra versión del positivismo según la cual la ciencia consiste en la acumulación de la experiencia observacional y su resumen en leyes interpretadas con cierto sabor instrumentalista, al modo de Mach, Duhem o Carnap. La imagen de esta ciencia sin lágrimas ni profundidad metafísica no revelaba una ontología de base que pudiese sufrir descalabros y discontinuidades revolucionarias. La reacción antipositivista, dirigida en Alemania por E. Husserl y en Francia por $\mathrm{H}$. Bergson (profesores ambos de Koyré) coincidió con una reacción neo-kantiana contra el materialismo y el positivismo, a la vez que subrayaba el idealismo trascendental que subordinaba

${ }^{3}$ Cf. The Essential Tension, The University of Chicago Press, 1977, pág. 11. 
la experiencia al pensamiento, sin reducirse a él. ${ }^{4}$ La idea fundamental es que la ciencia no es autosuficiente y debe fundarse filosóficamente en la estructura trascendental del sujeto. De ahí el alcance filosófico de estudiar su historia.

Buena parte de las variedades del kantismo surge del problema del carácter de la teoría trascendental y de la relación entre la realidad y las apariencias. Una respuesta a qué conocimiento podemos tener de los constituyentes a priori de la sensibilidad y el entendimiento consistió en tomarlos como conocimientos científicos del tipo de los de la psicología introspectiva (Fries) o incluso de la neurología (Helmholtz) o la biología (von Uexküll). Este tipo de respuesta "psicologista" fue criticada por Husserl y Frege, ya que con ella volvemos al empirismo y al escepticismo humeano. Sin embargo, retornar a una más firme observancia kantiana en términos de una deducción trascendental de las condiciones de posibilidad de la experiencia en general, al margen de los sujetos empíricos implicados, llevaba a una psicología trascendental a priori más opaca e insostenible que el psicologismo $u$ otra forma de naturalismo, por más abocado al escepticismo que estuviese.

Una salida más débil respecto a la fundamentación del conocimiento, pero que entrañaba un programa que se podía emprender, consistió en situarse in media res y considerar, al modo de Cohen y Natorp, que la filosofia trascendental es una reconstrucción del hecho de la ciencia. La tradición francesa encaja en esta tendencia de buscar la solución a problemas filosóficos acudiendo a la historia de la ciencia. Bergson, por ejemplo, consideraba que dicha historia poseía un meollo metafísico porque, al ser las ciencias un

4 La famosa idea de Koyré de los años cuarenta, según la cual la Revolución Científica no fue provocada por nuevos descubrimientos empíricos, sino por una revolución en las ideas filosóficas, concretamente el platonismo, se puede leer en los artículos de la antologia preparada por A. Beltrán y prologada por mí, Pensar la ciencia, Barcelona: Paidós, 1994. Sin embargo no es original suya, sino que se retrotrae a esta reacción antipositivista de principios de siglo, visible en E. Cassirer, Das Erkenntnisproblem in der Philosophie und Wissenschaft der neueren Zeit, 3 vols., Berlín, 1906, 1907, 1920; E.A. Burtt, The Metaphysical Foundations of Modern Science, Nueva York, 1924; A.N. Whitehead, Science and the Modern Worla, Nueva York, 1925. 
producto del entendimiento, reflejaban las limitaciones y características de su modo de operar en la imposición de racionalidad y orden a una realidad en perpetuo flujo. Del mismo modo $\mathrm{E}$. Boutroux, en De l'idée de loi naturelle (1895), había concebido las leyes científicas como compromisos engañosos que congelaban una realidad cambiante. Otro tanto puede encontrarse en la concepción dinámica de la razón de $\mathrm{G}$. Bachelard o en la razón constituyente de A. Lalande. La french connection de Kuhn procede de estos epistemólogos neokantianos, especialmente L. Brunschvicg y E. Meyerson, "cuya búsqueda de categorías cuasi-absolutas del pensamiento en las viejas ideas científicas ha producido brillantes análisis genéticos de conceptos que la tradición principal de la historia de la ciencia había malinterpretado o despreciado". Aunque su inclinación por estos autores se debe más a lo que vieron en los textos históricos que a su filosofía, muy exótica en el medio académico norteamericano, parecen constituir la principal fuente de su exposición a la problemática que, como decía, lo llevó a definirse como kantiano post-darwinista. Además, la lectura de estos autores, especialmente Identidad y realidad (1908) de Meyerson, data del período de formación en la Harvard Society of Fellows. La sugirió K.R. Popper en lo que constituyó la principal, si no única influencia del filósofo y caballero sobre el pensamiento de Kuhn. ${ }^{6}$

Tanto Meyerson como Brunschvicg presentan sus piezas históricas y su análisis de la ciencia en un formato kantiano. Así, por ejemplo, en L'idealisme contemporain (1905), Brunschvicg recurre a la historia de la ciencia porque ahí se manifiesta el movimiento objetivo del pensamiento que produce una aproximación a la realidad merced al diálogo interno de la mente en su lucha por vencer la diafonía entre los conceptos y entre éstos y la experiencia. Con la debida distancia, eso recuerda la tesis de Kuhn en su

5 The Essential Tension, pág. 108; cf. también, pág. 11.

6 Véase la entrevista con Kuhn en G. Borradori, The American Philosopher, The University of Chicago Press, 1994, pág. 159. La edición original italiana del libro, Coversazioni americane, G. Laterza, es de 1991. 
contribución al homenaje a Koyré, según la cual la función de los experimentos mentales es enseñar algo tanto acerca de los conceptos como acerca de la naturaleza, por lo que, aunque no presenten datos nuevos, se parecen más a la experimentación real de lo que hasta entonces se pensaba. ${ }^{7}$ Por su parte, el Meyerson de Identidad y realidad (1908) argumenta a favor de su idea de que la razón impone a una realidad irracional esquemas de identidad (como la causalidad) y trata de reducirla a espacio y geometría. El libro, no obstante lo peregrino de su idea de fondo, contiene una argumentación refinada mediante la historia de la ciencia para ilustrar la dialéctica entre los esquemas a priori identificadores y la resistencia de la realidad a esa eliminación de la diversidad y la temporalidad. Así, la "evacuación" del tiempo merced a la conservación de la materia y la energía y la reversibilidad mecánica se ve frustrada por el principio de Carnot que niega la resolución de la realidad en espacio indiferenciado y reclama la presencia de la línea temporal; o la reducción parmenídea del universo al espacio por mor de la relatividad, se frustra con la discontinuidad cuántica.

No es de extrañar el interés de Kuhn por el uso filosófico que hace Meyerson de la historia de la ciencia y el distanciamiento de su metafísica, en gran parte compartida por Koyré, de que la ciencia es el camino de la mente a la verdad a través de la dialéctica entre la realidad irreductible y la razón que trata de imponer la reducción a la geometría. Con todo, la idea de la existencia de marcos conceptuales que se imponen a la experiencia y la constituyen (la dependencia teórica de la observación) fue uno de los elementos básicos de la crítica antipositivista de Kuhn que presentaba así reminiscencias neokantianas. Sin embargo esta idea se modificó de dos maneras. La primera, inspirada por Koyré, disolvía el esquema global meyersoniano en una pluralidad de

7 "A Function for Thought Experiments", L'aventure de la science. Melanges Alexandre Koyré, Paris: Hermann, 1964, Vol. II: 307-34; ahora cap. 10 de The Essential Tension. 
ellos por mor de la discontinuidad entre los diferentes marcos conceptuales, lo que unido a una perspectiva darwinista del desarrollo de la ciencia (con la que termina La estructura), entrañaba negar que la ciencia se dirigiese hacia ningún objetivo, la verdad o la realidad. El itinerarium mentis in veritatem era una ilusión. En segundo lugar, la influencia de la lectura del libro de Fleck sobre la sífilis, titulado debidamente La génesis y desarrollo de un hecho científico (1935), influyó en su sociologización de los marcos a priori de la experiencia y en la eliminación de toda necesidad, dirección y esquema global en el desarrollo del conocimiento. Veremos ambas cosas en los dos apartados siguientes.

\section{La naturaleza de la discontinuidad}

Ya señalamos cómo la filosofía de la ciencia ingenua de Kuhn halló su camino a Damasco en el caluroso verano de 1947 cuando trataba de estudiar los antecedentes aristotélicos de la meçánica moderna de Galileo, paradigmáticamente estudiada por Koyré en sus Estudios galileanos (1939). Se convenció de la existencia "de un tipo global de cambio en el modo en que las personas ven la naturaleza y le aplican el lenguaje, que no se puede describir con propiedad diciendo que consta de añadidos al conocimiento o de meras correcciones parciales de errores". 8 Cuatro años antes, Koyré había escrito en una vena similar que "lo que los fundadores de la ciencia moderna [...] tuvieron que hacer no fue criticar y combatir ciertas teorías erróneas para corregirlas y sustituirlas por otras mejores; tenian que hacer algo muy distinto. Tenían que destruir un mundo y sustituirlo por otro. Tenían que remodelar y reformar sus conceptos, tenían que desarrollar una nueva manera de ver el Ser, un nuevo concepto del conocimiento, un nuevo concepto

- The Essential Tension, pág. xiii. 
de ciencia". ${ }^{9}$ En ambos casr se propone una concepción holista de las teorías científicas comu construcciones del mundo, que torna anacrónico separar y proyectar sobre ellas lo que retrospectivamente es un error o un adelanto respecto al estado actual del conocimiento.

Koyré era un emigrado ruso en París como Meyerson, con el que se reunía semanalmente para mantener discusiones filosóficas y con el que compartía la visión metafísica de la historia de la ciencia como la imposición de una visión platónica a la realidad (la buena ciencia se hace a priori, era una de sus afirmaciones predilectas), "persiguiendo siempre el sueño grandioso e insensato de reductione scientiae ad geometriam, esto es, del Ser al espacio, del Ens al Non-Ens". No obstante no es ese el objetivo fundamental de los trabajos de Koyré, quien confiesa no haberse mantenido fiel a los planes de Meyerson, "pues en mis trabajos me he entregado sobre todo a mostrar, no el fondo idéntico del pensamiento humano, sino las diferencias de sus estructuras en las diversas épocas de su historia". ${ }^{10}$ En esto fue un maestro y como tal lo tiene Kuhn por lo que respecta a la historia de la ciencia ${ }^{17}$ Koyré resulta difícilmente igualable a la hora de presentar en toda su riqueza de matices la unidad de cada sistema pretérito de pensamiento, reuniendo los aspectos técnicos, filosóficos, metafísicos y culturales en un todo orgánico que nos permite ver desde dentro la manera de pensar de Cusa, Boehme o Kepler.

La manera que tiene Koyré de estudiar los sistemas científicos como si fueran sistemas filosóficos, en los que la discontinuidad y no acumulación de conocimientos es patente, tiene sus raíces, como decía, en la reacción anti-positivista francesa de principios de siglo. El objetivo para la historia de la ciencia era descubrir un elemento teórico con el que se pudieran explicar las características

9 "Galileo and Plato", Journal of the History of Ideas, 4 (1943): 400-28; ahora en Estudios de Historia de la Ciencia, Madrid: Siglo XXI, 1977, pág. 155.

10 "Message d'Alexandre Koyré à l'occasion du centenaire de la naissance d'Emile Meyerson", Bulletin de la Societé française de Philosophie, 53 (1961): 115-6.

11 Cf. The Essential Tension, pág. 21. 
de las teorías del pasado, tanto su estructura con sus aciertos y "errores", como la estructura de las inferencias y el sistema de valores en la toma de decisiones. En este sentido A. Tannery hablaba del "estado de espíritu contemporáneo", mientras que Lévy-Bruhl recurría a las "mentalidades" y Koyré, a la "estructura de pensamiento". Del mismo modo que en filosofía de la ciencia se reaccionaba frente a la concepción del conocimiento científico como ristras de hechos convencionalmente resumidos en leyes $y$, por el contrario, se prestaba atención a las ontologías implicadas por la ciencia para dar cuenta de la heuristica y de los nexos entre los fenómenos, en la historia de la ciencia se recurría al concepto teórico de cultura, mentalidad o estructura del conocimiento para dar sentido al conjunto de la ciencia del pasado, "errores" incluidos. Estas entidades, expresadas muchas veces de manera fluvial, como "las grandes corrientes" de Boutroux o los "vastos ríos espirituales" de Koyré, trataban de cumplir una función en la explicación histórica a pesar de su vaguedad. Así, A. Rey urgía el paso de la historia de fechas y hazañas heroicas a la historia de la civilización que explicase tales hechos. En el caso de la ciencia, el factor clave era "el espíritu filosófico de la representación del universo". H. Metzger importaba a la historia de la ciencia el concepto de "mentalidad" de su tío para buscar "la estructura del espíritu humano". L. Febvre, por su parte, recomendaba "recomponer el material mental de los hombres de una época; reconstruir todo el universo físico, intelectual y moral de una generación". ${ }^{12}$ De este transfondo proviene la tendencia al holismo de Koyré y la identificación de la filosofía como el núcleo de esas estructuras de ideas que definen una perspectiva científica.

La novedad de Kuhn consistió, sin embargo, en ofrecer una teoría de en qué consiste el meollo de la discontinuidad más

12 Cf., v.g., A. Koyré, La philosophie de Böhme, París: Vrin, 1929, pág. 508; A. Rey, "Histoire de la science ou des sciences", Archeion, 12 (1930): 1-4; H. Metzger, "La philosophie de Lucien Lévy-Bruhl et l'histoire des sciences", Archeion, 12 (1930): 15-25, pág. 23; L. Febvre, "Un chapitre de l'histoire de l'esprit humain", Reoue de Synthèse historique, 43 (1927): 37-60, pág. 56. 
precisa empíricamente y menos metafísica. No discutiré aquí este aspecto bien conocido de la filosofía de Kuhn. En su contribución, Fernando Broncano examina algunas críticas como las de D. Davidson y $P$. Kitcher al relativismo de la inconmensurabilidad e intraducibilidad (pero véase también el artículo de Julio César Armero) y sitúa la buena historia de la ciencia en el descubrimiento de las prácticas perdidas que están fuera del lenguaje, detrás de la manera de encadenar las piezas del mismo a la naturaleza. Por su lado, Eloy Rada plantea el problema del carácter metahistórico de conceptos kuhnianos como revolución, ciencia normal o paradigma, que pertenecerían al esquema historiográfico a priori más que a la propia historia, con lo que Kuhn sería primariamente un filósofo que desarrolla sus categorías en relación con la historia de la ciencia, al modo de Meyerson y Koyré.

Como hemos visto, la idea crucial de Kuhn en su segunda ruptura a comienzos de 1959 fue la de que la comunidad de reacciones y decisiones frente a la actividad de resolución de rompecabezas en que consiste la ciencia se debe al hecho de compartir ejemplos paradigmáticos de soluciones relevantes y no creencias o conceptos formulados lingüísticamente. Como señala Kuhn, "surgieron dificultades cuando traté de especificar el consenso enumerando los elementos acerca de los cuales se suponía que concordaban los miembros de una comunidad dada. Para explicar la manera en que realizaban la investigación y especialmente para explicar la unanimidad con la que de ordinario evaluaban las investigaciones de los demás, tenía que atribuirles un acuerdo acerca de las características definitorias de términos cuasi-teóricos como "fuerza" y "masa", "mezcla" y "compuesto". Pero la experiencia como científico y como historiador sugería que dichas definiciones rara vez se enseñaban y que los ocasionales intentos de formularlas despertaban con frecuencia un desacuerdo acusado. Aparentemente no existía el consenso que buscaba, aunque no podía escribir sin él el capítulo sobre la ciencia 
normal". ${ }^{13}$ La solución mediante los paradigmas en sentido original restringido (no la matriz disciplinar) fue la conocida solución de Kuhn.

Esta idea, frente a la hidrología francesa, poseía dos características. La primera es que resultaba más concreta y empíricamente identificable que las mentalidades. La segunda, que entrañaba una naturalización debida al carácter tácito (psicológico) y comunitario (sociológico) de la operación de los paradigmas. El carácter tácito del acuerdo paradigmático entrañaba un enfoque cognitivista de la cuestión, planteando los a priori de la investigación científica en términos psicológicos. Kuhn exploró esta vía durante tres lustros, hasta "Second Thoughts on Paradigms" (1974), antes de dar un giro hacia un planteamiento predominantemente lingüístico. La indagación libre que promovía la pertenencia a la Society of Fellows (1948-51) lo condujo a estudiar la psicología de la Forma, muy presente en La estructura con las metáforas de cambios de paradigma como conmutaciones gestálticas, así como a Piaget, por lo que respecta a las etapas de construcción del mundo de los niños. Los diferentes mundos en que viven niños y científicos, descubiertos a finales de los cuarenta, desempeñaron una función central en el pensamiento inicial de Kuhn. "He adquirido en parte mi conocimiento acerca de cómo plantear preguntas a los científicos muertos examinando cómo interroga Piaget a los niños vivos. Recuerdo vivamente de qué manera estuvo presente esa influencia en mi primer encuentro con Alexandre Koyré, la persona que ha sido mi maitre más que cualquier otra. Le dije que había aprendido de los niños de Piaget a comprender la física de Aristóteles. Su respuesta en el sentido de que había sido la física de Aristóteles la que le había enseñado a entender a los niños de Piaget no hizo más que confirmar mi impresión acerca de la importancia de lo que había aprendido". ${ }^{14}$ La inversión de Koyré se compadece muy bien con

\footnotetext{
13 The Essential Tension, pág. xviii y sig.

14 "Les notions de causalité dans le développement de la physique" (1971); ahora en The Essential Tension, pág. 21 y sig.
} 
su anti-psicologismo y la subordinación de los procedimientos científicos a las ideas filosóficas, como veremos enseguida.

Tuvo ocasión de proseguir la exploración de los aspectos psicológicos del funcionamiento paradigmático de la ciencia en el Center de Stanford (1958-9), donde coincidió con representantes notables de la corriente de la New Look que daría paso a la psicología cognitiva, como G.A. Miller y K. Pribram que estaban a punto de publicar con E. Galanter una obra crucial, Planes y estructura de la conducta (1960). Proponían en ella un nuevo enfoque de la conducta mediante el símil del ordenador, con el que jugó Kuhn en los años setenta para modelar el procesamiento de la información sensorial bajo paradigmas. ${ }^{15}$ Una idea básica tomada de la New Look es la del procesamiento de arriba abajo, que encuentra acogida en el capítulo $X$ de La estructura (pág. 113, nota 2) sobre "las revoluciones como cambios en la visión del mundo" para justificar la función a priori de los paradigmas en la experiencia perceptiva. ${ }^{16}$

Sin embargo, Kuhn simultaneó el enfoque psicológico de los a priori con el lingüístico, que terminó predominando. ${ }^{17}$ Ya en su estancia en la Society of Fellows, que le facilitó el tránsito de la física a la historia y la filosofía, había descubierto los trabajos de B.L. Whorf y su famosa tesis de cómo el lenguaje posee efectos causales sobre la visión del mundo. También leyó a Quine, con quien

15 Aparecen menciones al programa de ordenador en "Reflections on my Critics" (1970), en I. Lakatos y A. Musgrave, Criticism and the Growth of Knowledge, Cambridge, 1970, pág. 274, y algo más explícitamente en "Second Thoughts" (1974), ahora capítulo 12 de The Essential Tension.

16 Hemos discutido las implicaciones psicológicas de la filosofía kuhniana en C. Solís y P. Soto, "Kuhn y la ciencia cognitiva", en C. Solís (ed.), Alta tensión: historia, filosofía y sociologia de la ciencia. Ensayos en memoria de Thomas S. Kuhn, Barcelona: Paidós (en prensa).

17 En su entrevista con Borradori, págs. 161 y 166, Kuhn define su evolución como "un creciente hincapié en el lenguaje, que desempeña cada vez más la función que tenian en La estructura de las revoluciones científicas los cambios gestálticos. En ese primer libro se habla mucho de cambio de significado. Ahora hablo más de términos con referencia, dotando a cada uno de ellos de una taxonomía. Incluso interpreto cada vez más como cambio taxonómico el que tiene que ver con la inconmensurabilidad". 
coincidió una década más tarde en el Center de Stanford. "Dos dogmas del empirismo" (1953) le enseñó a entender cómo determinadas leyes tienen el carácter de cuasi-empíricas aunque a priori y se retiran de la contrastación. Asimismo, Quine le enseñó a evitar plantear el problema de la inconmensurabilidad en términos de cambio del significado, considerando que los científicos que trabajan en tradiciones diversas "hablan lenguajes diferentes, lenguajes que expresan distintos compromisos cognitivos adecuados a mundos diferentes". ${ }^{18}$ Quine es el principal y casi el único pensador anglosajón que influyó sobre Kuhn. ${ }^{19}$ La contribución de Ulises Moulines a este homenaje dilucida en qué sentido la ciencia empírica precisa un marco a priori (de naturaleza histórica) y ofrece una caracterización precisa y profunda de las intuiciones kuhnianas acerca del caracter híbrido de los conceptos y leyes esenciales de una teoría, mostrando con ello cómo la historia de la ciencia puede beneficiarse de la filosofía de la ciencia.

Como ya es de sobra sabido, el elemento fundamental en el anti-positivismo de Kuhn es la posibilidad de ligar directamente al mundo un lenguaje complejo (un sistema taxonómico o léxico) sin necesidad de recurrir a un lenguaje neutral de observación en

${ }^{18}$ The Essential Tension, págs. xxii y sig.; $186 \mathrm{n} .9$, y 258 donde se cita a Whorf y a Quine sobre el contenido legal de los conceptos que muestran una mezcla imposible de desentrañar de lo analítico y sintético, de la definición y la ley.

19 Algunas veces de manera negativa, como en el problema de la traducción radical que Kuhn obvia mediante la exigencia de que el historiador y los científicos separados por paradigmas se tornen bilingues más que traductores. Otros autores anglosayones presentes en las doctrinas de Kuhn fueron Putnam, con quien polemizó respecto a la teoría causal de la referencia y la verdad. En el comienzo de su formación cita a Lovejoy y Butterfield, muy influido como él mismo por Koyré en lo que respecta a la hístoria de las ideas científicas. Pese a su insistencia, Borradori (op. cit., págs. 157-8 y 165) no logró que Kuhn reconociese deuda alguna con la tradición pragmatista americana que confiesa no haber leído. Las discusiones con Quine y Putnam se producen sobre todo al elaborar su tesis de madurez según la cual los cuerpos pasados de creencias se especifican por disponer de diferentes léxicos. Cf. "Possible Worlds in History of Science", en S. Allen (ed.), Possible Worlds in Humanities, Arts, and Sciences, Berlín, 1988: 9-32, y "Dubbing and Redubbing: The Vulnerability of Rigid Designation", en C. Wade Savage (ed.), Scientific Theories, University of Minnesota Press, 1990: 298-318. 
cuyos términos se especificaría el léxico por la vía de definiciones reductivas o reglas de correspondencia. Los paradigmas suministran un modelo de procesamiento capaz de explicar cómo es posible hacerlo psicológicamente, pasando después a centrarse en las propiedades del léxico para dilucidar la inconmensurabilidad local y la discontinuidad científica.

En el resumen que ofreció Kuhn del libro que le ocupó la última década de su vida, "The Road Since Structure" (1991), señala que el problema central de sus preocupaciones filosóficas gira en torno a la inconmensurabilidad, problema que aborda ahora exclusivamente en términos lingüísticos, dejando de lado los planteamientos psicológicos ligados a la Gestalt. Pero asimismo el planteamiento del cambio de teoría como aprendizaje de un lenguaje nuevo resulta demasiado general, debiendo restringirse al cambio de significado de un tipo de términos, los taxonómicos o de clases naturales o artificiales. En su contribución a este homenaje, "Objetos y propiedades", Anastasio Alemán discute el problema quineano general del alcance ontológico del lenguaje, problema que Kuhn planteó para cada uno de los lenguajes locales de las teorias científicas. Pero mientras que Quine, y con él Kuhn, limita el importe ontológico a objetos y clases que satisfacen un criterio de identificación o individuación, Alemán argumenta con precisión la conveniencia de incluir también la existencia de propiedades. Por su lado, Julio Armero analiza más en concreto "los argumentos lingüísticos de Kuhn" exponiendo una cierta tosquedad filosófica por parte de Kuhn, así como la inadecuación de sus interpretaciones de la traducción radical, la inescrutabilidad de la referencia y el carácter del principio de caridad tanto en la traducción como en la interpretación que constituye el caballo de batalla del historiador. (Véanse también las consideraciones de Fernando Broncano.) Las precisiones de Armero deberian ser tomadas muy en cuenta en toda dilucidación del carácter y alcance de las últimas propuestas léxicas de Kuhn, que constituyen el meollo de sus tesis sobre el relativismo, la racionalidad, el realismo y la verdad. 
Sin embargo falta aún por reconocer un elemento fundamental y de consecuencias de gran alcance en la filosofia de Kuhn, cual es la perspectiva sociológica. Cuatro páginas más arriba señalábamos una diferencia crucial con su maître en historia de la ciencia, Alexandre Koyré, que podriamos diagnosticar como naturalismo frente a idealismo. Koyré, cuya defensa de la historia de la ciencia interna podria tildarse más bien de idealismo, ${ }^{20}$ se oponia a toda teoría que reconociese alguna eficacia causal sobre el desarrollo de la ciencia a factores no ideales (filosóficos y aun religiosos), como los intereses tecnológicos, sociológicos, políticos y económicos. Era parte de la oposición heredada de Husserl a toda forma de "psicologismo" que chocaba frontalmente con las tendencias naturalizadoras de Kuhn. El antinaturalismo en general y antimaterialismo en particular llevaron a Koyré a extremos extravagantes, como cuando rechazaba la incidencia del problema práctico de la determinación de la longitud en el mar sobre el desarrollo de los cronómetros marinos. Como se quejaba $\mathrm{H}$. Guerlac, todo intento de contextualizar la ciencia en aspectos más amplios que los puramente ideales presentes en la sociedad, se encontraba en el mejor de los casos con la descalificación "intéressant, mais un peu marxiste".

Después de la guerra, la influencia de Koyré sobre la naciente generación de historiadores de la ciencia anglosajones fue tal que oscureció los trabajos de R.K. Merton, por no hablar de los enfoques marxistas del tipo de los de B. Hessen. En 1963, A.R. Hall $^{21}$ diagnosticaba la muerte de la incipiente sociología de la ciencia a manos del internismo koyreano. Desde que diez años antes aparecieran en Centaurus unos artículos sobre las relaciones sociales de la ciencia, nada más se había publicado en las revistas especializadas, como Isis, Annals of Science, Revue d'Histoire des

20 Véase mi introducción, "Alexandre Koyré y la Historia de la Ciencia" a la selección de escritos de A. Koyré preparada por Antonio Beltrán, Pensar la ciencia, Barcelona: Paidós, 1994, especialmente 30-39.

21 "Merton Revisited, or Science and Society in the Seventeenth Century", History of Science, 2 (1963): 1-16. 
Sciences o Archives Internationales. Cuando Hall escribía estas cosas, acababa de publicarse La estructura, donde Kuhn identificaba los a priori de la investigación de la ciencia normal con la psicología y la sociología de las comunidades científicas, sin reconocer un tribunal superior en la toma de decisiones. ${ }^{22}$ El "psicologismo" o naturalismo era rampante. Basta hacer hoy la "prueba de Hall", consistente en repasar las revistas del gremio, para constatar el triunfo arrasador del sociologismo contra el intelectualismo idealista del maitre. Con toda su admiración por la recuperación que hacía Koyré de los sistemas de creencia del pasado, Kuhn disolvió la unidad del "espíritu humano" en su itinerario hacia la verdad en la pluralidad de marcos a priori de la experiencia enraizados en la "naturaleza" psico-social del funcionamiento de las diversas comunidades. La metafísica de Meyerson y el idealismo filosófico de Koyré se disolvieron en la sociología de grupos humanos históricos. Y la génesis de esta perspectiva kuhniana se encuentra, una vez más, en el pensamiento continental europeo, esta vez en la obra de Fleck descubierta también por serendipia a mediados de siglo, cuando era Junior Fellow de la Society of Fellows de Harvard.

\section{La epistemología socializada}

Kuhn aprendió de Koyré a concebir la historia de la ciencia como un proceso discontinuo basado en la asunción de marcos previos a la medición y experimentación. Fleck le enseñó a cambiar el sujeto de la historia, sustituyendo al individuo por el colectivo de especialistas. Fleck escribió su monografía La génesis y el desarrollo de un hecho científico (1935) como una crítica al positivis-

\footnotetext{
22 "El reconocimiento de la existencia de un grupo profesional exclusivamente competente y la aceptación de su función como arbitro de los logros profesionales posee ulteriores implicaciones. Los miembros del grupo [...] han de tenerse por los únicos poseedores de las reglas de juego u otro fundamento equivalente de la univocidad de los juicios". (The Structure, pág. 168)
} 
mo lógico del Círculo de Viena, con el que estaba familiarizado por su participación en el grupo de Lwów-Varsovia en el que se hallaban discípulos de Twardowski, como Ajdukiewicz, Kotarbiński y Łukasiewicz. La idea básica que critica Fleck es la del Aufbau, consistente en concebir el conocimiento como una construcción lógica precisa a partir de bloques firmes de la experiencia. Por el contrario, los informes básicos no están libres de supuestos y son producto de una construcción social. Kuhn ha reconocido que una de las cosas que le enseñó Fleck fue a ver cómo los "hechos" poseen un ciclo vital de desarrollo, tranquilizándolo acerca de la adecuación de su propia manera de concebir la dependencia teórica de los hechos que estaba formulando con ayuda de la psicología de la Gestalt. ${ }^{23}$ La otra, que dicha construcción posee un carácter colectivo o social.

El intento del positivismo de reformar la herencia kantiana, poniendo la aportación del mundo externo en lo dado a la experiencia y la del sujeto, en la lógica vacía de contenido, resulta ingenua porque la experiencia está ya estructurada. Además esa estructuración viene dada por una cultura colectiva, "la tradición, la formación y la costumbre dan origen a una disposición a percibir y actuar conforme a un estilo, es decir, de forma dirigida y restringi$d a "{ }^{24}$ Una de las virtudes del libro de Fleck es que sus ideas se exponen al hilo del análisis de cómo se realiza la investigación en el laboratorio, extraído de su experiencia como médico y microbiólogo. Su trabajo es sociológico también en el sentido de atender al contexto heuristico más que al lógico: "es prácticamente imposible establecer enunciados protocolares basados en una observación directa, de los que se sigan los resultados como una conclusión lógica. Algo así sólo es posible en la legitimación ulterior de un saber, pero no mientras se está haciendo el trabajo propiamente cognoscitivo" (Id., pág. 136). Los hechos científicos son pues "una

23 Cf. su prólogo a la edición inglesa del libro de Fleck, Genesis and Development of a Scientific Fact, The University of Chicago Press, 1979, pág. ix.

24 Traducción española, La génesis y desarrollo de un hecho cientifico, Madrid: Alianza, 1986, pág. 131. 
creación artificial y que sólo tiene una mera relación genética tanto con respecto al propósito original como al contenido de la 'primera' observación" (Ibidem). El marco previo de esta construcción de la experiencia proviene no de las categorias de un sujeto trascendental, sino de las condiciones psico-sociales de los grupos de investigación históricamente dados. "La actitud disciplinada de un colectivo, conformada a un estilo y mantenida durante generaciones, produce una 'imagen real' del mismo modo que la actitud febril produce una alucinación" (pág. 158n). La psychologie des foules (1895) de G. Le Bon ofrece un modelo de la creación colectiva de la 'realidad'. "Se pinta demasiado nítidamente la línea divisoria entre lo pensado y lo existente. Al pensamiento le debe ser reconocido un cierto poder para crear objetos y a los objetos, su procedencia del pensamiento; mejor dicho, del pensamiento de un colectivo conformado por un estilo" (pág. 159n; las cursivas son mías). Es incluso demasiado fácil ver el paralelismo del colectivo y del estilo con las comunidades científicas y los paradigmas (esta vez en sentido amplio de matriz disciplinar) de Kuhn, así como el efecto que poseen sobre la construcción social de la experiencia y del mundo fenoménico.

Las condiciones a priori de la experiencia quedan así naturalizadas y relativizadas a colectivos sociales dotados históricamente de un 'estilo de pensamiento' que no es sino "un percibir dirigido con la correspondiente elaboración intelectiva y objetiva de lo percibido" (pág. 145). La relativización de la construcción a priori de la realidad entraña la relatividad de la verdad: "no se puede decir nunca que el mismo pensamiento es verdadero para A y falso para B. Si A y $B$ pertenecen al mismo colectivo de pensamiento, entonces el pensamiento es verdadero o falso para ambos. Pero si pertenecen a colectivos distintos, entonces ya no se trata realmente del mismo pensamiento [...]. La verdad no es convención, sino que vista con perspectiva histórica, es un suceso en la historia del pensamiento $y$, dentro de su contexto momentáneo, es una coerción del pensamiento marcada por el estilo" (pág. 146 y sig.). El resultado de esa coerción no es una manera específica de 'interpretar' la experiencia, sino 
que constituye directamente la experiencia: "se adquiere la capacidad de percibir inmediatamente un sentido, una forma (Gestalt), una unidad cerrada. Al mismo tiempo, desde luego, se pierde la capacidad de ver cualquier cosa que contradiga dicha forma. Es justamente dicha disposición para el percibir dirigido lo que constituye el componente principal del estilo de pensamiento" (pág. 139). Paralelamente, el estilo afecta a los significados lingüísticos que, con el paso del tiempo y las transformaciones culturales, dan lugar a cambios inconmensurables, ${ }^{25}$ de manera que "cuando el estilo de pensamiento está muy alejado del nuestro, ya no es posible su comprensión, pues las palabras no pueden traducirse y los conceptos no tienen nada en común con los nuestros" (pág. 190). Sin embargo, esas estructuras históricas discontinuas e inconmensurables coaccionan de tal modo el pensamiento que lo convierten en "conocimiento objetivo y aparentemente real" (pág. 192). Los análisis históricos de la concepción del 'fósforo' en la Naturphilosophie (págs. 175-81) o de las representaciones gráficas en anatomía (págs. 181-90) rellenan de sustancia una doctrina que, cantada a cappella, pudiera parecer un tanto ruda. En esto también se parecen Fleck y Kuhn.

Las semejanzas entre ambos puntos de vista contienen también un elemento post-kantiano y significan naturalizar el carácter del elemento a priori del conocimiento. Kuhn señala que entre las coincidencias de sus perspectivas está que "los efectos de la participación en un colectivo de pensamiento son de algún modo categóricos o a priori. Lo que suministra a sus miembros el colectivo de pensamiento es algo similar a las categorías kantianas, pre-requisito de cualquier pensamiento en absoluto. La autoridad de un colectivo de pensamiento es así más cuasi-lógica que social, si bien sólo existe para el individuo en virtud de su inducción en un grupo". ${ }^{26}$ Ahora bien, las categorías léxicas (en las que el

25 El término polaco usado por Fleck en 1927 es, gústenos o no, niewspólmiernośc. Cf. el prólogo de L. Schäfer y T. Schnelle en la traducción española, pág. 20.

26 Prólogo a la edición inglesa citada, pág. ix. 
último Kuhn pone el elemento a priori) no son como las kantianas. "El léxico suministra las precondiciones de la experiencia posible, como las categorías kantianas. Pero, frente a lo que ocurría con sus predecesoras kantianas, las categorias léxicas pueden cambiar y cambian tanto con el tiempo como con el paso de una comunidad a otra".27

Pero no todo cambia. La sociologización kuhniana está muy alejada de la de sus sedicentes seguidores posmodernos, como los adictos al programa fuerte. La diferencia estriba en que Kuhn no está dispuesto a desconectar la ciencia de la epistemología, disolviéndola en puras modas y convenciones sociales. Mientras que los sociologistas estiman que los procedimientos científicos se subordinan a la defensa de intereses sociales parciales de los grupos, más que a la verdad o a la resolución efectiva de rompecabezas, para Kuhn no es esa la función de la sociología en la epistemología científica, como señala Fernando Broncano en el último apartado de su artículo. La sociología es relevante para caracterizar los colectivos de comunicación improblemática por compartir determinados compromisos o conocimientos a priori que, por inconmensurables que sean en algunos lugares, generan resultados objetivos. Para Kuhn la objetividad de la ciencia está anclada en la continuidad por lo que respecta a la resolución de rompecabezas y a los valores que la caracterizan como actividad colectiva, mientras que los kuhnianos posmodernos consideran que todos los valores cambian de comunidad a comunidad y de época a época. ${ }^{28}$ La perspectiva darwinista de Kuhn lo lleva a considerar la resolución de problemas como lo que ejerce la selección de las propuestas de los sujetos y en ese sentido es realista. No realista respecto a que las categorías léxicas propuestas correspondan con la cosa en sí, sino en el sentido de que ésta ejerce una resistencia a las propuestas teóricas. El mundo "es plenamente sólido; no es en lo más mínimo respetuoso con los deseos y

2 "The Road since Structure", loc. cit. en la nota 1, pág. 12.

28 The Essential Tension, pág. xxi y sig. 
apetencias del observa lor. [...] ¿Acaso un mundo que cambia con el tiempo y de una comunidad a la siguiente puede corresponder a lo que en general se alude como 'el mundo real'? No veo cómo se le puede negar el derecho a ese título. Suministra el medio, el escenario de toda vida individual y social. Impone restricciones rígidas a dicha vida; la conservación de la existencia depende de adaptarse a ellas; $y$ en el mundo moderno la actividad científica se ha convertido en una herramienta primaria para la adaptación. ¿Qué más se le puede razonablemente pedir a un mundo real?"29 El mundo dependiente de las categorías históricas, el mundo fenoménico, no es el único, aunque así parezca desde una ciencia normal. En efecto, en la ciencia extraordinaria se modifica tan sólo la parte puesta por el sujeto y no el mundo en sí: "subyacente a todos esos procesos de diferenciación y cambio ha de haber, por supuesto, algo permanente, fijo y estable, aunque como la Ding-ansich de Kant, sea inefable, indescriptible, indiscutible ${ }^{130}$

En qué medida el deseo kuhniano de objetividad, realismo y verdad científica es consistente con su análisis psico-sociológico de los procedimientos científicos, en qué medida se logra de este modo disipar el peligro de relativismo e irracionalidad, es un problema que aún dará trabajo a los filósofos durante algún tiempo.

Otro de esos problemas lo constituyen las ciencias sociales. La impostación sociológica de la filosofía kuhniana de la ciencia ha despertado multitud de análisis orientados a dilucidar la naturaleza y estado de desarrollo de las propias ciencias humanas comparadas con las naturales. Amparo Gómez analiza los diferentes diagnósticos sobre el carácter de la sociología y la economía en función de cómo se entiende el concepto de 'paradigma', y apunta que la convivencia multiparadigmática correspondería al carácter plural de la sociedad. La diversidad de los intereses y puntos de vista de los grupos sociales sería una de las fuentes de

20 "The Road", pág. 10.

30 Id., pág. 12. 
la inalcanzada unanimidad propia de la 'ciencia natural normal' caracterizada por Kuhn. Francisco Álvarez, por su parte, incide en el mismo problema al conectar la imagen kuhniana de la ciencia, basada en decisiones sociológicas de las comunidades de especialistas y no en algoritmos generales accesibles a un sujeto epistemológico abstracto (sin intereses sociales), con el giro retórico que consiste en reconocer la necesidad constante de tomar decisiones en condiciones de incertidumbre. La presencia de argumentaciones no euclídeas suscitadas por necesidades prácticas insoslayables lleva a tomar en serio a la retórica y a enfrentarse a las consecuencias que ello entraña para la idea de racionalidad.

Hemos subrayado la influencia europea sobre el pensamiento de Kuhn que contrasta con su desconocimiento de la tradición norteamericana. Ya aludimos a su negativa a reconocer deuda alguna con los pragmatistas a pesar de las sugerencias de Borradori. Sin embargo, su pensamiento tiene conexiones con el pragmatismo, especialmente dado el papel central que tiene la resolución práctica de problemas en la selección de teorías ${ }^{31}$ y en la concepción de la verdad a la que no desea renunciar. ${ }^{32}$ Además, la ambigüedad de las doctrinas de Kuhn respecto al realismo y al subjetivismo son las propias del pragmatismo. Por ejemplo, Peirce favorecía un naturalismo darwinista de la verdad como característica de determinados hábitos de acción capaces de alcanzar un

${ }^{31}$ "Si tuviera que escribir de nuevo La estructura de las revoluciones científicas haria mucho más hincapié en la noción de resolución de rompecabezas. A los científicos se los entrena para resolver rompecabezas y hay una considerable ideología en todo ello. Tiene que haber más y más rompecabezas, soluciones más y más precisas a rompecabezas anteriores. Por tanto hay una finalidad". Borradori, 1994, pág. 167.

32 "Ciertamente creo en la referencialidad del lenguaje. Siempre hay algo sobre el encaje referencial implicado en la experiencia que dice si se usa para hacer enunciados verdaderos o falsos. Hay un sentido, un sentido profundo, en el que creo absolutamente en la teoría de la verdad como correspondencia". Id. pág. 166. 
equilibrio homeostático con la experiencia objetiva, de modo que a la larga las taxonomías y leyes científicas (los generales) son reales, independientemente de la mente (8: 13). Pero el pragmatismo también daba para posturas más escépticas, típicas de William James cuando extendía lo práxico más allá de lo empírico hasta justificar la aceptabilidad pragmática de la religión, dada su utilidad para producir resignación y paz en espíritus caquécticos. Frente a la objetividad de los "generales" de Peirce, las leyes de James son constructos nominalistas tendientes más a la conveniencia de intereses que a la correspondencia de la verdad. En el extremo, al comienzo de sus Studies in Humanism (1909), F.C.S. Schiller llevaba la volubilidad de la verdad hasta negar todo viso de correspondencia y afirmar que decir que una proposición es verdadera equivale sencillamente a aceptar que "promueve nuestros fines". La realidad (: lo que hay es lo que dicen que hay nuestras teorias garantizadas pragmatistamente) resulta tan mutable como las teorías que promueven intereses. La variedad de kuhnianismos no parece muy distinta de la variedad de pragmatismos. De alguna manera el pragmatismo y sus problemas debía de estar en el ambiente de Harvard en que se formó Kuhn y, ciertamente, en el estilo filosófico de Quine y Putnam con quienes interactuó frecuentemente.

Así pues, una peculiar tradición europea nos ha sido devuelta a todos después de su reelaboración en un contexto cultural exótico como el americano. La contribución a este volumen de Francisco Zamora es un excelente estudio de la introducción de Kuhn en una España que empezaba a emerger de un inacabable y casposo período fascista y en la que la filosofía se debatía entre un dogmatismo metafísico periclitado y un envaramiento neopositivista cuya utilidad iba poco más allá de apoyar la negativa a comulgar con ruedas de molino. Entonces Kuhn representaba para muchos la combinación de la precisión de la filosofía de la ciencia con la flexibilidad y pluralidad de la historia. Sin embargo, y con algunas excepciones como la de José Romo Feito, las traducciones de Kuhn al español (tanto de España como de 
México) han sido muchas veces traicioneras y casi siempre inelegantes. La última contribución debida a Antonio Beltrán critica los desmanes más sobresalientes de este aspecto esencial de la transmisión de Kuhn en España.

Tuve ocasión de conocer a Kuhn en sus últimos años de Princeton, poco antes de que se recluyera a escribir un libro largamente meditado, como todos los suyos, pero desgraciadamente no acabado. Habiendo presenciado su manera incansable y aguda de argumentar, sé que este volumen de homenaje a su memoria, a pesar de la calidad de las contribuciones, no hace plena justicia a la magnitud de su esfuerzo por ver el fondo profundo de la ciencia tras la imagen superficial y sin lágrimas del positivismo y los manuales.

He organizado este homenaje en memoria de Kuhn gracias a una sugerencia de E. Rada y J. Echeverría, en nombre de Éndoxa y de la Sociedad Española de Lógica y Filosofía de la Ciencia. Inicialmente todas las contribuciones iban a ver la luz en Éndoxa. No obstante, la respuesta de los colegas fue tan considerable y variada que surgió la posibilidad de publicar en una editorial comercial los artículos más populares, esto es, los capaces de llamar la atención de un público más amplio que el profesional. El libro Alta tensión: historia, filosofía y sociología de la ciencia. Ensayos en memoria de Thomas $S$. Kuhn reúne una quincena de contribuciones y será publicado este otoño por la editorial Paidós. A un año de la muerte de Kuhn, deseamos adelantarnos a rendir tributo desde Éndoxa a la persona que más ha hecho este siglo por explicar a los no científicos (como queria Conant), no menos que a los propios científicos, en qué consiste esa actividad cognoscitiva y práctica que, desde las intuiciones visionarias del siglo XVII hasta hoy, se ha integrado de tal modo en la cultura humana que forma ya parte inextricable de su constitución. 